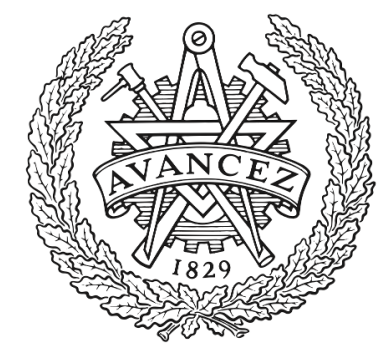

\title{
CHALMERS
}

UNIVERSITY OF TECHNOLOGY

\section{Numerical analysis of the influence of natural convection in attics - A CFD analysis}

Downloaded from: https://research.chalmers.se, 2023-04-26 04:07 UTC

Citation for the original published paper (version of record):

Shankar, V., Bengtson, A., Fransson, V. et al (2018). Numerical analysis of the influence of natural convection in attics - A CFD analysis. AIP Conference Proceedings, 1978.

http://dx.doi.org/10.1063/1.5044080

N.B. When citing this work, cite the original published paper. 


\title{
Numerical Analysis of the Influence of Natural Convection in Attics - A CFD Analysis
}

\author{
Vijay Shankar ${ }^{1, \text { a) }}$, Andreas Bengston ${ }^{2}$, Victor Fransson ${ }^{2}$, Carl-Eric Hagentoft ${ }^{2}$ \\ ${ }^{1}$ Luleå University of Technology, Luleå, Sweden \\ ${ }^{2}$ Chalmers University of Technology, Maskingränd 2, 41258 Göteborg, Sweden \\ a) vijay.shankar@ltu.se
}

\begin{abstract}
As the design of the building envelope has changed, the parameters that govern thermal insulation, air tightness and the complex heat transfer processes are often neglected. This aim of this research work is to numerically investigate the influence of natural convection in attics with joists and insulation. With the help of Computational Fluid Dynamics (CFD), more specifically the software ANSYS Fluent, 3D simulations are conducted for both porous layer and air cavity to estimate the magnitude of heat transfer. The same was executed for porous media with two different values of permeability. A suitable turbulence model has been applied to account for the highly sensitive buoyancy driven flow. The results are presented in form of dimensionless numbers, Nusselt number versus modified Rayleigh number. It can be concluded that the temperature difference and permeability play a vital role.
\end{abstract}

Keywords — computational fluid dynamics (CFD), Heat Transfer, Building physics, Fluid mechanics

\section{INTRODUCTION}

The heat transfer processes are often the limiting factor when designing energy efficient buildings for sustainable development. By investigating the heat flows and losses combined with the air movement in buildings, a greater understanding and knowledge of the same can be obtained. Computational Fluid Dynamics (CFD) is a great tool in order to examine these phenomena and to apply this knowledge in the process of designing energy efficient and sustainable buildings. When compared to real-life experiments, CFD simulations have several advantages. It is a more convenient and cost-effective method, since expensive experimental equipment is not needed. In this research work, the heat transfer in a principal cold attic has been numerically investigated by using the commercial CFD software ANSYS Fluent. The attic model consists of a horizontal layer of insulation with an overlying region of air. The attic model can be viewed in cross section in figure. 1, along with its cross-sectional dimensions. Besides these dimensions, the attic is $1.8 \mathrm{~m}$ deep, the insulation has a maximum thickness of $0.4 \mathrm{~m}$, and the angle of the roof is $20^{\circ}$. At two sides of the insulation, wind deflectors are placed. They have the same angle as the roof, and are situated there to prevent the ventilation system to force air to flow directly into the insulation.

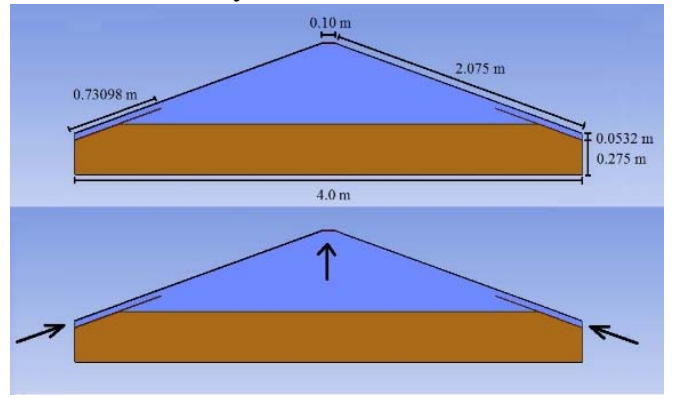

FIGURE 1. The attic model, seen from the side. The upper region represents the air cavity and the lower one the porous insulation. The upper picture shows the dimensions of the principle attic model, and the lower picture visually represents the inlet and outlet of the ventilation system.

The presence of a ventilation system is however not taken into account in this work. The model also includes the presence of wooden joists which are located within the insulation, see figure. 2 . The joists are assumed to have a 
significant impact on the overall heat transfer in the attic. The distance between the middle of two joists is $0.6 \mathrm{~m}$, and the joists have a width of $0.045 \mathrm{~m}$ and a height of $0.135 \mathrm{~m}$. Each joist reaches from one side of the attic along the floor to the other side. The computational mesh for this research work consisted of 503390 cells and was constructed using only hexahedronal cells in the software Ansys ICEM. Simulations were performed with a constant temperature at the bottom of the attic,

Thot, of $294 \mathrm{~K}$, and varying, lower, temperatures at the boundary of the roof of the same, $\mathrm{T}_{\text {cold. }}$ The temperature difference was varied between 10 to $50 \mathrm{~K}$. Two different types of insulation with different values of permeability were investigated, in order to make a comparison and analyze the impact of the permeability. The applied virtual model for simulating the heat transfer in a medium containing both porous media as well as an air cavity with help of CFD has been developed and verified by the authors in a previous work [1]. This model is in turn based on previous research [2-9] and verified by comparison to experimental work performed by [10].

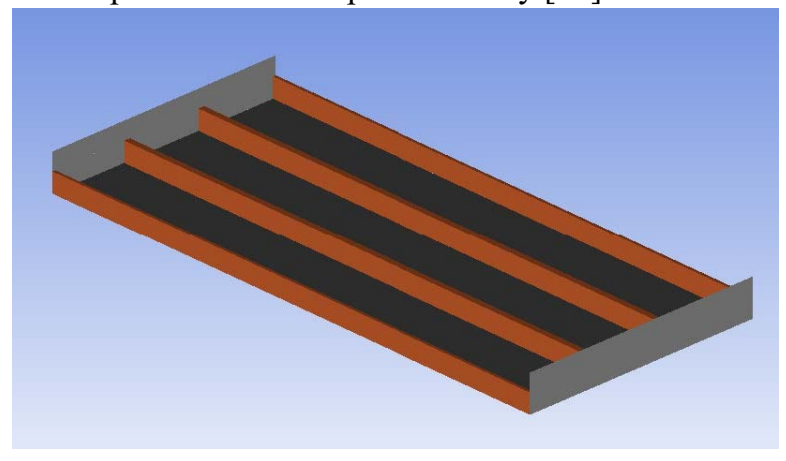

FIGURE 2. View over the wooden joists seen from above.

\section{NUMERICAL SETUP}

The numerical setup for the simulations in this project was exactly the same as in the previous work in Shankar, Bengtson and Fransson (2014). The applied boundary conditions for the attic were, with respect to the view in figure. $1, \mathrm{~T}=\mathrm{T}_{\text {cold }}$ on the roof and the sides that are in contact with the cold air, $\mathrm{T}=\mathrm{T}_{\text {hot }}$ on the bottom of the attic and $\mathrm{q}=0$ on the vertical boundaries of the insulation. In addition to this, symmetry boundary conditions were applied to the sides of the attic that are parallel to the joists longitudinal direction. This was done in order to simulate that the attic continues in this direction rather than being closed by walls.

\section{RESULTS}

The Nusselt number was computed using the same methodology as in Shankar, Bengtson and Fransson (2014). Computations were executed for nine temperature differences for each permeability, thus, the total number of simulations was eighteen. Due to the limited amount of space, the scales for both the temperature and vector plots can be quite hard to read. For the temperature contour plots in figure. 4 the scale in each picture goes from 294 to 294 minus the temperature difference, where red is the warmest (bottom surface) and blue the coldest (top surface). For the velocity vector plots in figure. 5, figure. 6 the exact velocities are not of interest, but they are in the order of $10^{-4}$ $\mathrm{m} / \mathrm{s}$ in the insulation and $10-2 \mathrm{~m} / \mathrm{s}$ in the air cavity. The results in figure. 4 , figure. 5 were taken in a plane parallel to the joists longitudinal direction in the middle of the attic, whereas the results in figure. 6 were taken in a plane perpendicular to the joists longitudinal direction in the middle of the attic. 


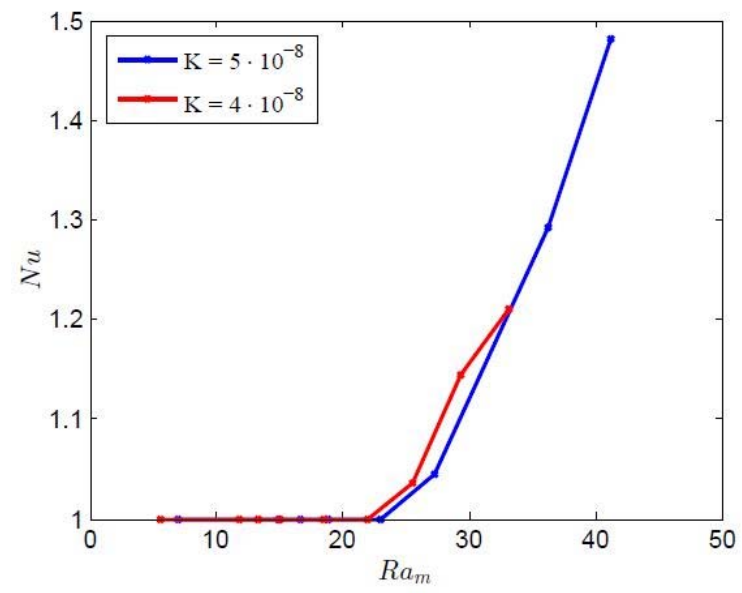

FIGURE 3. Plot of The Nusselt number versus the modified Reynolds number for the cases with permeability of $5 * 10^{-8} \mathrm{~m}^{2}(\mathrm{blue}$ line) and the case with permeability of $4 * 10^{-8} \mathrm{~m}^{2}$
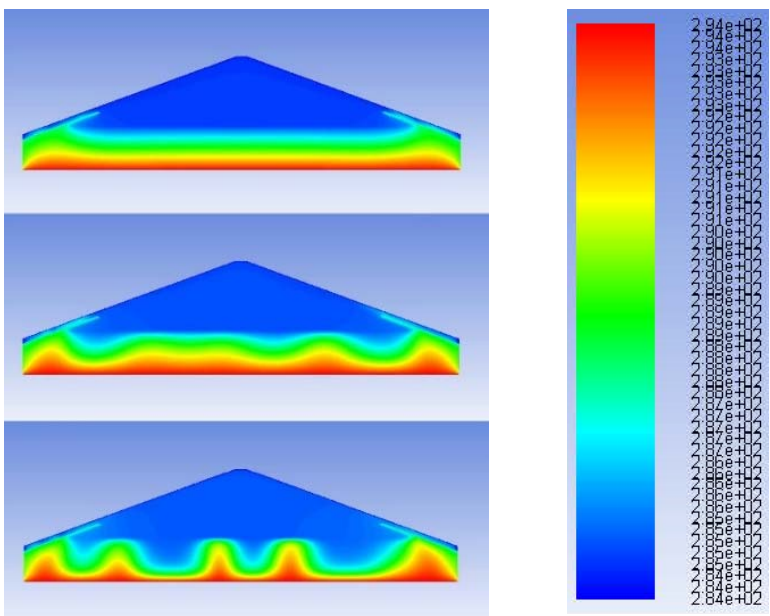

FIGURE 4. Temperature contours for the case with permeability of $4 * 10^{-8} \mathrm{~m}^{2}$ visualized in a plane parallel to the joists located in the middle of the domain. The plots are for, in order from above, 10, 35 and $50 \mathrm{~K}$ temperature difference.
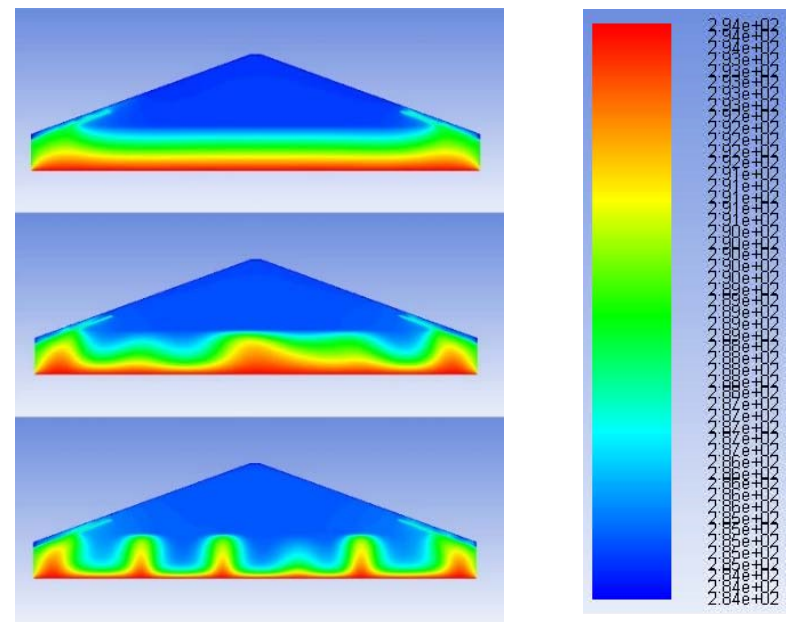

FIGURE 5. Temperature contours for the case with permeability of $5 * 10^{-8} \mathrm{~m}^{2}$ visualized in a plane parallel to the joists located in the middle of the domain. The plots are for, in order from above, 10, 35 and $50 \mathrm{~K}$ temperature difference. 


\section{DISCUSSIONS}

As seen in figure. 3, the simulated results of the Nusselt number versus the modified Rayleigh number shows a physical and reasonable behavior. As the temperature difference increases, the magnitude of heat transfer due to convection in the insulation also increases. The onset of convection occurs a approximately $\operatorname{Ra}_{\mathrm{m}^{*}}=22$. Looking at the temperature contour plots in, figure. 4 it can be concluded that there is no real tendencies in the pictures as the temperature difference increases. All the temperature profiles looks unsymmetrical and chaotic except for the first picture in both figure. 4 this is because for this temperature difference convection has not yet started so all the heat is transported by conduction and radiation only. These mechanisms of heat transfer transports heat equal in all directions, which explains why those pictures looks symmetric.

The velocity profiles taken along the long side of the attic, figure. 5,6 looks perhaps a bit counter intuitive, as it would be expected to see the hotter air rising upwards, and not downwards or in the direction out from the paper. This behavior can be more explained by looking at the temperature profiles taken across the long side of the attic, figure. 7,8. These figures shows that the air above the insulation basically flows across the domain. This indicates that it seems like the flow will tend to orient itself in one big cylindrical convection cell across the domain. The impact from the joists on the heat transfer is exemplified in figure.10. It is expected, since the joists conducts heat better than the insulation, that the flow should be accelerated upwards over the joists. This happens over some of the joists in this actual plane, while the air also flows down over some of the joists. This is probably due to the overall air flow in the domain, which in some situations forces the air to flow down over the joists. The joists has, however, a clear impact on the heat and air flow. Natural convection is known to be an highly unstable phenomena in nature, and can for example easily be affected by small changes in geometry and boundary conditions. An example of this can be seen when studying the both pictures in figure. 6 . As the temperature difference increases from 35 to $50 \mathrm{~K}$, the direction of the flow has changed to the opposite direction. In order to ensure the accuracy of the simulations, the residuals of the simulations were investigated.

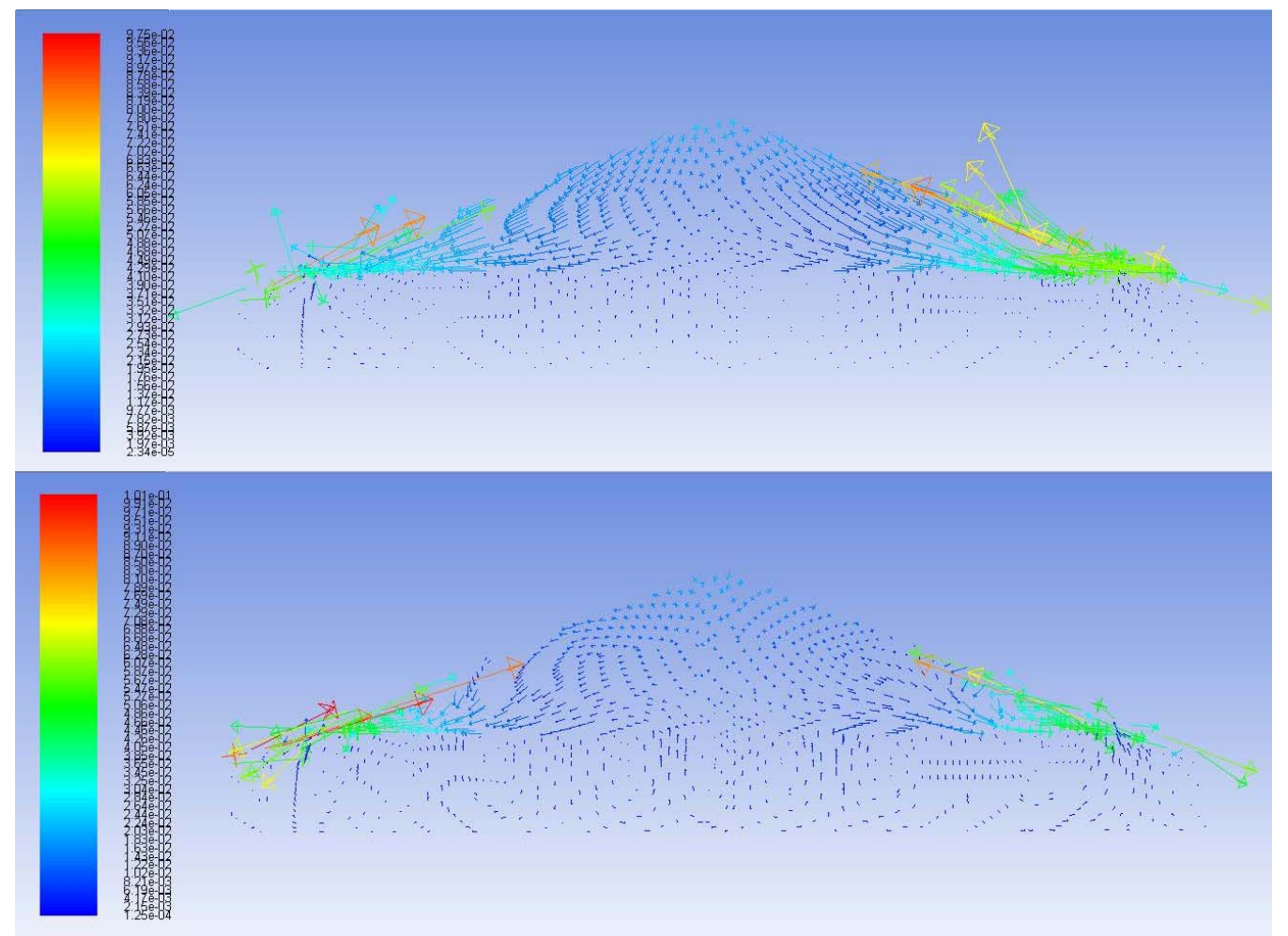

FIGURE 6. Velocity vectors for the temperature difference of, in order from above, 35 respectively $50 \mathrm{~K}$, for the permeability of $4 * 10-8 \mathrm{~m} 2$. The plots are visualized in a plane parallel to the joists located in the middle of the domain. 
The residuals of a simulation with temperature difference of $35 \mathrm{~K}$ can be seen in figure. 10 , the residuals in this figure are representative for the residuals obtained in all the performed simulations. Generally, the values for the different residuals are good and they pass the general criterion for convergence which is recommended by Fluent ANSYS, Inc. (2011). This is true for all the residuals except continuity, which being in the order of 10-1, unfortunately is really high in all simulations. It is interesting to compare the results obtained in this research work to the results obtained by the authors in the previous work, performed with the same settings in Fluent but for a cuboid box Shankar, Bengtson and Fransson (2014). In that case, the graphical results for both the temperature and the velocity were almost exclusively symmetrical and had some clear tendencies that developed when the temperature difference was increased. This indicates that in the case with the box, steady state solutions were more easily obtained than in this case. The values of the residuals, figure.9, indicates the same thing, as especially the values for continuity were a lot higher than in the previous work.

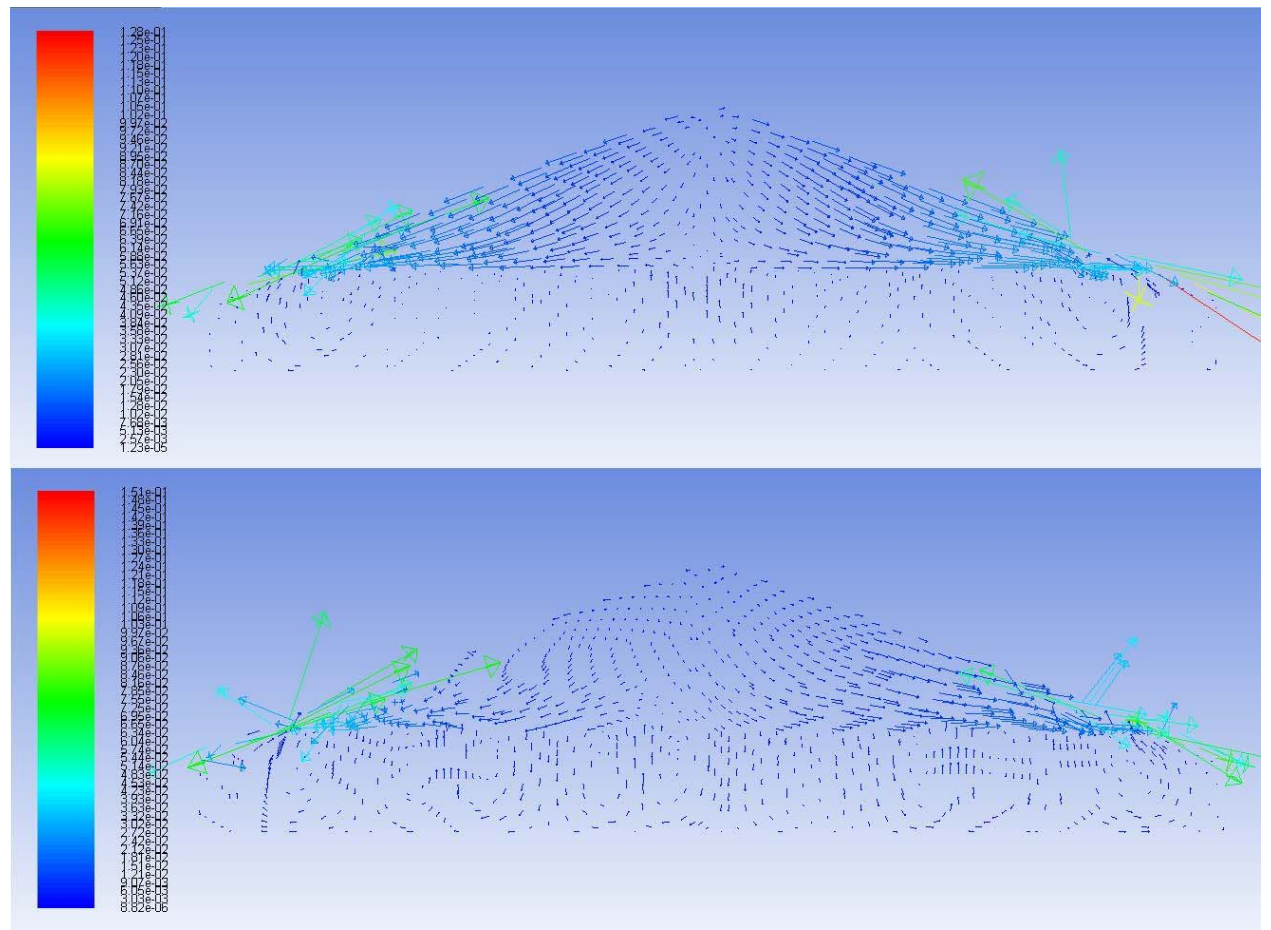

FIGURE 7. Velocity vectors for the temperature difference of, in order from above, 35 respectively $50 \mathrm{~K}$, for the permeability of $5 * 10^{-8} \mathrm{~m}^{2}$. The plots are visualized in a plane parallel to the joists located in the middle of the domain.

It would have been interesting to perform simulations on a more dense mesh, i.e. a mesh with more computational cells, to see if that would make the continuity residuals go down. This was however not possible to try, since the academic license of Fluent that was used restricts the amount of computational cells to 512000. It would also be interesting to perform time dependent, so called unsteady, simulations for this case. This could maybe help preventing the high residuals for continuity, although very large computational resources would be demanded in order to perform simulations for a realistic period of time. 


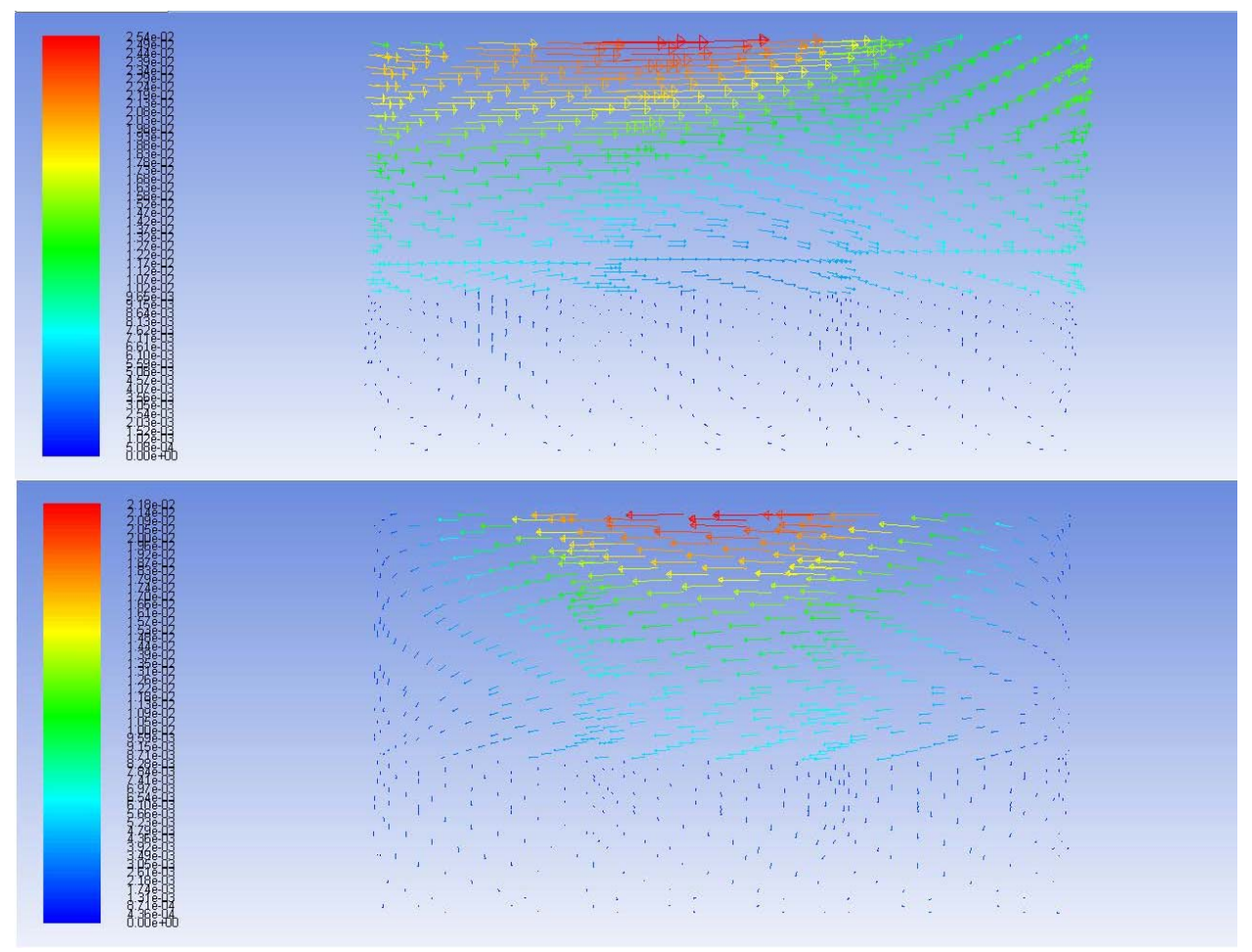

FIGURE 8. Velocity vectors for the temperature difference of, in order from above, 35 respectively $50 \mathrm{~K}$, for the permeability of $4 * 10^{-8} \mathrm{~m}^{2}$. The plots are visualized in a plane perpendicular to the joists located in the middle of the domain.

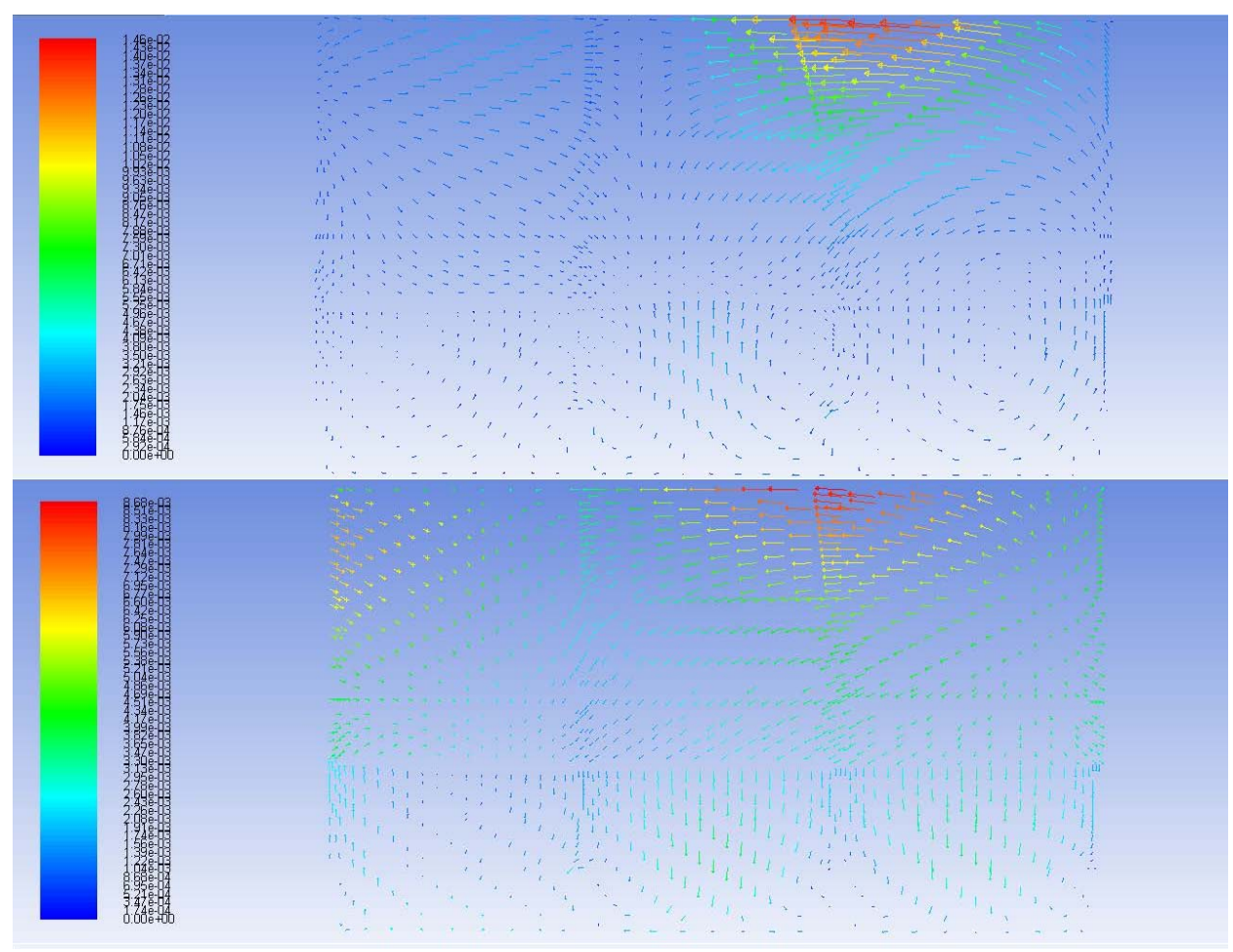

FIGURE 9. Velocity vectors for the temperature difference of, in order from above, 35 respectively $50 \mathrm{~K}$, for the permeability of $5 * 10^{-8} \mathrm{~m}^{2}$. The plots are visualized in a plane perpendicular to the joists located in the middle of the domain. 


\section{CONCLUSION}

The applied method and model for the simulation of an attic containing porous insulation, air cavity and wooden joists gives satisfactory and realistic results. The results obtained from the simulations presented in this article shows a physical behavior both from a visual perspective (figures for temperature and velocity distribution), and from a heat transfer perspective (Figure. 3). The methodology provided in Shankar, Bengtson and Fransson (2014) transfers well to the case involving a cold attic with joists. The simulations suffer from high residuals in continuity. Simulations on a more dense mesh and unsteady simulations instead of steady could maybe make the residuals go down.

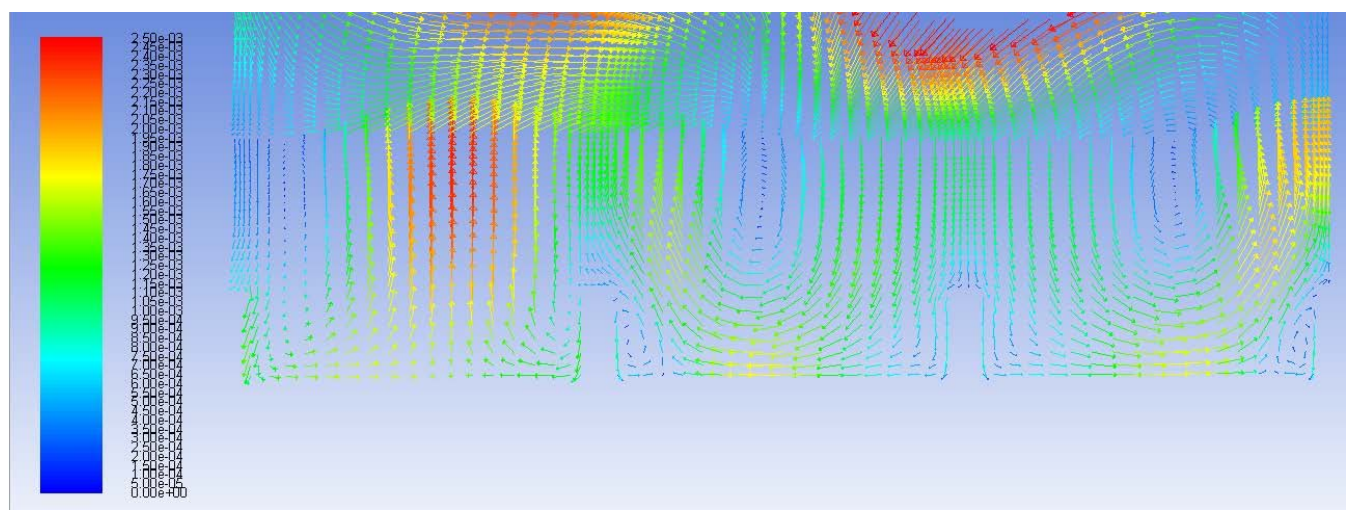

FIGURE 10. Velocity vectors scaled to show the velocities in the insulation for the temperature difference of $40 \mathrm{~K}$ and permeability of $4 * 10^{-8} \mathrm{~m}^{2}$. The figure is visualized in a plane perpendicular to the joists located in the middle of the domain.

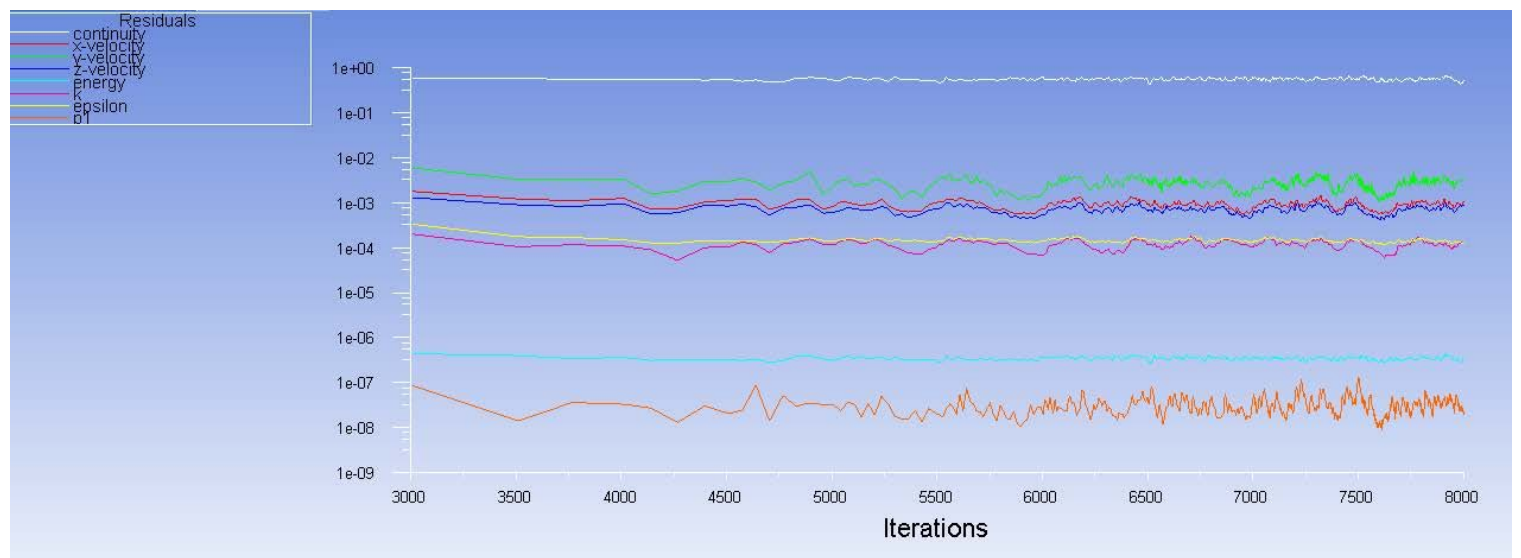

FIGURE 11. Computational residuals for a simulation with temperature difference of $35 \mathrm{~K}$ and permeability of $5 * 10^{-8} \mathrm{~m}^{2}$.

\section{FUTURE WORK}

In order to get a more accurate model of a cold attic, the ventilation of the attic also has to be taken into account. This should be the first step going forward with this research. Since natural convection is naturally an unstable and time dependent phenomenon, it would also be interesting to perform unsteady simulations on the attic model.

\section{ACKNOWLEDGEMENTS}

This research work has been conducted with the help of financial and computer resources obtained from Swedish Energy Agency, Luleå University of Technology and the Division of Building Technology, Chalmers University of Technology. 


\section{REFERENCES}

[1] Shankar V, Bengtson A and Fransson V (2014) Influence of Heat Transfer Processes in Porous Media with Air Cavity - A CFD Analysis., Accepted for publication in the 8th International Conference on Computational and Experimental Methods in Multiphase and Complex Flow, Valencia, Spain, 20-22 April,2015.

[2] Delmas A and Wilkes K (1992) Numerical analysis of heat transfer by conduction and natural convection in loose-fill fiber glass insulation effects of convection on thermal insulation.,ORNL/CON-338.

[3] Delmas A and Arquis E (1995) Early initiation of natural convection in an opened porous layer due to the presence of solid conductive inclusions., Journal of Heat and Mass Transfer, 117.

[4] Shankar V and Hagentoft C.E. (1999) Numerical convection in insulating porous medium., Indoor air 99, Edinburgh.

[5] Shankar V and Hagentoft C.E. (1999) Influence of natural convection on the thermal properties of insulating porous medium with air cavity., Indoor air 99, Edinburgh.

[6] Shankar V and Hagentoft C.E. (2000) Numerical investigation of natural convection in horizontal porous media heated from below comparisons with experiments., ASME International, Pittsburgh.

[7] Shankar V, Davidson L and Olsson E (1995) Numerical investigation of turbulent plumes in both ambient and stratified surroundings., Journal of INDOOR AIR, Denmark.

[8] Shankar V, Davidson L and Olsson E (1992) Ventilation by displacement: Calculation of flow in vertical plumes., ROOM VENT, Aalborg, Denmark.

[9] Wahlgren P (2001) Convection in Loose-fill Attic Insulation., PhD Thesis, Chalmers University of Technology, Gteborg.

[10] Serkitjis M (1995) Natural convection heat transfer in a horizontal thermal insulation layer underlying an air layer., PhD Thesis, Chalmers University of Technology, Gteborg.

[11] ANSYS, Inc. (2011) ANSYS FLUENT Users Guide, ANSYS, Inc, Canonsburg, latest edition. 